\title{
Modeling of a Leg and Knee System for the Analysis of Human Gait by Means of State Feedback Control
}

\author{
P. A. Ospina-Henao ${ }^{\mathrm{a}, 1}$, César H. Valencia ${ }^{\mathrm{b}}$, Marcelo Becker ${ }^{\mathrm{c}}$, Zuly A. Mora P b, \\ and S. M. Vásquez ${ }^{\mathrm{b}}$ \\ ${ }^{a}$ Departamento de Ciencias Básicas, Universidad Santo Tomás. Carrera 18 No. 9 - 27. \\ PBX: (+57)76985858. Bucaramanga. Colombia. \\ ${ }^{\mathrm{b}}$ Facultad de Ingeniería Mecatrónica, Universidad Santo Tomás. Carrera 18 No. 9 - \\ 27. PBX: (+57)76985858. Bucaramanga. Colombia. \\ ${ }^{\mathrm{c}}$ Departamento de Engenharia Mecânica (SEM), University of São Paulo (USP), PBX: \\ (+55)1633738646. São Carlos-SP. Brazil.
}

\begin{abstract}
This paper presents the modeling, simulation and control of a human gait system, which consists of the modeling of a leg by means of Euler's classical mechanics and Lagrange's formalism, where the equations of motion of the joint are obtained both of the hip as of the knee and the solution of these. In addition, a state feedback control was implemented and the controller gains were determined by means of the Ackerman formula, based on the equations of motion rewritten in state space and simulated in simulink, where the behavior of the system can be observed with control.
\end{abstract}

Keywords. Control, Feedback of States, Formalism Euler - Lagrange, Human Gait.

\section{Introduction}

Human gait is a complex process that integrates the relationship of various subsystems of the human body necessary to generate biped movement. In addition, this locomotion process is marked by some phases, which are the support phase and the equilibrium phase that is selected in a series of stages that begin with the heel contact with the ground and end with the takeoff of the foot [1]. This research seeks to provide a dynamic model that describes the movement of the leg joints such as the hip and knee using the EulerLagrange formalism and a control, which serves as a support to reproduce human gait in an electromechanical environment. The objective was to investigate and describe a series of basic mechanical and physiological mechanisms behind human walking. The methodologies used were the biomechanical analysis of movement and physiology [2]. It is important to note that one leg dynamics is only one of many possible approaches to its study. The pathological approach integrates simple principles, clinical observations, and

\footnotetext{
${ }^{1}$ Corresponding Author: Departamento de Ciencias Básicas, Universidad Santo Tomás. Carrera 18 No. 9 27. PBX: (+57) 76985858. Bucaramanga. Colombia. E-mail: paolo.ospina@ustabuca.edu.co
} 
controlled experiments performed to explain the advantages of the method. The dynamic study and control by state feeding that we present here show how the dynamic walking approach could be applied to integrative study of gait pathologies [3]. These examples can be seen as starting points for new research, where experimental evidence will be indispensable [4]-[5]. Simple principles can't explain everything of the complexities of the march, but can help ask helpful questions what you have to answer to understand those complexities. There are two approaches to solving the direct and inverse dynamic model. A direct dynamic model is one that expresses the temporal evolution of joint coordinates as a function of the forces and torques involved, and an inverse dynamic model is one that expresses the forces and torques that intervene as a function of the evolution of joint coordinates and their derivatives [6]-[7].

It should be noted that the data obtained from this study is based on a simulation that uses computational tools. The simulation is based on a mathematical model that indicates a change between potential energy and kinetic energy, resembling the action of a double pendulum. This, with the evolution of this type of models in the future, they will be usable in the clinical context either for the training of medical personnel or to plan adjustments in the alignments that individuals may undergo, without presenting risks to patient stability [8]. The gait model produced with Solidworks was of great help in simulating the behavior of the leg system [9].

A model of the double pendulum in two dimensions is the pendulum-like motion of the swinging leg. The same conservation of mechanical energy is applied, so little work is necessary to move the swinging leg. Given a suitable starting position and velocity, the entire single limb support phase can be produced largely through the movement of 2 coupled pendulums representing the posture and swing of the leg. This modeling approach suggests that both the hip and the legs can take advantage of the pendulum dynamics during single-limb support [10] - [11].

During the present work several aspects of study were taken into account, starting in the section 2, by the dynamic modeling of the system through the Euler-Lagrange equations in a in a non-conservative way, the numerical model, the matrix representation of states and the graphic analysis of the numerical solutions were carried out.

Next, in section 3, the control of the dynamic system is shown, a rigorous study of the controllability and observability of the system is carried out, as well as the dominant poles and the gain matrix that allows designing the controller are calculated. The simulation of the human walking system is performed by applying the controller to an input signal. In the section 4 , we present the conclusions of the work.

\section{Symbolic model}

In clinical routine, gait analysis identifies normal or pathological movements. From a simplified model of the structure of the human body, perform this analysis at different levels and complementary techniques that evaluate different aspects of neuromuscular function, the mechanical model is described below. From Figure 1, we can take the following measurements: $x_{1}$ and $y_{1}$ are the coordinates of the center of mass of the upper leg (femur) and in the same way, $x_{2}$ and $y_{2}$ are the coordinates of the center of mass of the lower leg (tibia and fibula).

We can start with taking some measurements from the drawing, 


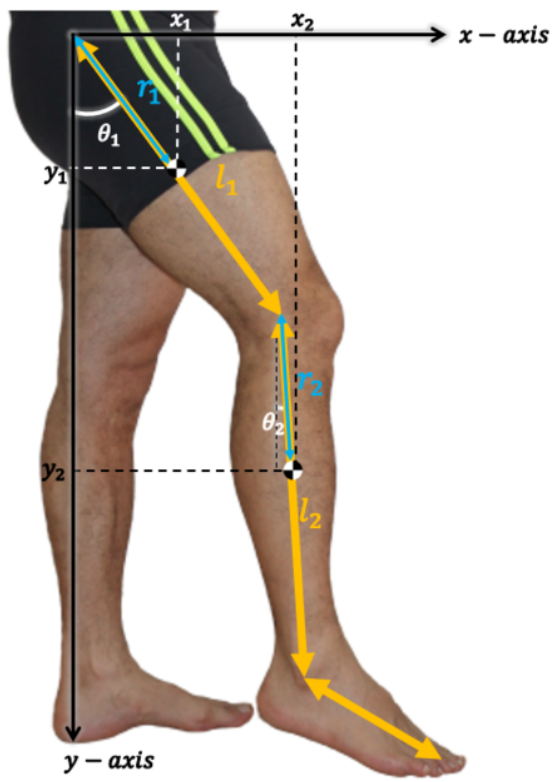

Figure 1. Initial system image

then we obtain the linear speed of the first link or the first joint,

$$
v_{1}^{2}=\dot{x}_{1}^{2}+\dot{y}_{1}^{2}=r_{1}^{2} \dot{\theta}_{1}^{2},
$$

and also, the linear speed of the second link,

$$
v_{2}^{2}=\dot{x}_{2}^{2}+\dot{y}_{2}^{2}=l_{1}^{2} \dot{\theta}_{1}^{2}+r_{2}^{2} \dot{\theta}_{2}^{2}+2 l_{1} r_{2} \dot{\theta}_{1} \dot{\theta}_{2} \cos \left(\theta_{1}-\theta_{2}\right),
$$

\subsection{Kinetic energy}

Kinetic energy is defined as

$$
K=\frac{1}{2} m_{1} v_{1}^{2}+\frac{1}{2} m_{2} v_{2}^{2},
$$

replacing Eqs. (1) and (2), in the previous expression we obtain,

$$
K=\frac{1}{2} m_{1} r_{1}^{2} \dot{\theta}_{1}^{2}+\frac{1}{2} m_{2}\left[l_{1}^{2} \dot{\theta}_{1}^{2}+r_{2}^{2} \dot{\theta}_{2}^{2}+2 l_{1} r_{2} \dot{\theta}_{1} \dot{\theta}_{2} \cos \left(\theta_{1}-\theta_{2}\right)\right] .
$$

\subsection{Gravitational potential energy}

Gravitational potential energy is defined as

$$
U=m_{1} g y_{1}+m_{2} g y_{2}
$$

getting,

$$
U=-m_{1} g r_{1} \cos \theta_{1}-m_{2} g l_{1} \cos \theta_{1}-m_{2} g r_{2} \cos \theta_{2}
$$




\subsection{Lagrangian density and equations of motion}

Lagrangian density is defined [12],

$$
L=K-U
$$

replacing Eqs. (3) and (4), in the previous expression we obtain

$$
\begin{aligned}
L & =\frac{1}{2} m_{1} r_{1}^{2} \dot{\theta}_{1}^{2}+\frac{1}{2} m_{2}\left[l_{1}^{2} \dot{\theta}_{1}^{2}+r_{2}^{2} \dot{\theta}_{2}^{2}+2 l_{1} r_{2} \dot{\theta}_{1} \dot{\theta}_{2} \cos \left(\theta_{1}-\theta_{2}\right)\right]+m_{1} g r_{1} \cos \theta_{1}+ \\
& +m_{2} g l_{1} \cos \theta_{1}+m_{2} g r_{2} \cos \theta_{2} .
\end{aligned}
$$

The Euler-Lagrange equations of motion have the form,

$$
\frac{d}{d t}\left(\frac{\partial L}{\partial \dot{\theta}_{i}}\right)-\frac{\partial L}{\partial \theta_{i}}+\frac{\partial D_{i}}{\partial \dot{\theta}_{i}}=\tau_{i}, \quad i=1,2 .
$$

here, $D_{i}$ is the Rayleigh dissipation function and it is only considered the viscous case [13], in other words, the friction, we can define Rayleigh function as a homogeneous quadratic form at generalized speeds, this results as a theoretical suggestion and they could be calculated in practice. With this in mind, Rayleigh's dissipation function can take the form,

$$
f_{r}=\frac{\partial D_{i}}{\partial \dot{q}_{i}}=\frac{1}{2} \beta_{i} \dot{q}_{i}^{2}
$$

From it, we can define the forces acting on the system, we must remember that the friction forces are proportional to a power of the instantaneous velocity, $f_{r}=-b v^{n}$. In our case, these friction forces are related to the forces delivered to each link in the system. For our case, we have two generalized coordinates that are $\left(\theta_{1}, \theta_{2}\right)$, resulting in the following equations,

resulting the motion equations,

$$
\begin{aligned}
& \tau_{1}=\ddot{\theta}_{1}\left(m_{1} r_{1}^{2}+m_{2} l_{1}^{2}\right)+m_{2} l_{1} r_{2} \ddot{\theta}_{2} \cos \left(\theta_{1}-\theta_{2}\right)+m_{2} l_{1} r_{2} \dot{\theta}_{2}^{2} \sin \left(\theta_{1}-\theta_{2}\right)+ \\
& +\left(m_{1} r_{1}+m_{2} l_{1}\right) g \sin \theta_{1}+\beta_{1} \dot{\theta}_{1} . \\
& \tau_{2}=\ddot{\theta}_{1} m_{2} l_{1} r_{2} \cos \left(\theta_{1}-\theta_{2}\right)+m_{2} r_{2}^{2} \ddot{\theta}_{2}-m_{2} l_{1} r_{2} \dot{\theta}_{1}^{2} \sin \left(\theta_{1}-\theta_{2}\right)+ \\
& \quad+m_{2} r_{2} g \sin \theta_{2}+\beta_{2} \dot{\theta}_{2} .
\end{aligned}
$$

\subsection{Numerical model}

To define the numerical model, the constant values are replaced, 
Table 1. System variables.

\begin{tabular}{|l|l|l|}
\hline Variable & Value & Units \\
\hline$m_{1}$ & 8 & {$[\mathrm{~kg}]$} \\
$m_{2}$ & 3.72 & {$[\mathrm{~kg}]$} \\
$l_{1}$ & 0.45 & {$[\mathrm{~m}]$} \\
$r_{1}$ & 0.195 & {$[\mathrm{~m}]$} \\
$r_{2}$ & 0.165 & {$[\mathrm{~m}]$} \\
$g$ & 9.81 & {$\left[\mathrm{~m} / \mathrm{s}^{2}\right]$} \\
$\beta_{1}$ & 2.288 & {$[\mathrm{~kg} / \mathrm{s}]$} \\
$\beta_{2}$ & 0.175 & {$[\mathrm{~kg} / \mathrm{s}]$} \\
\hline
\end{tabular}

here, $m_{1}$ is thigh mass, $m_{2}$ the calf mass, $l_{1}$ thigh length, $r_{1}$ length to center of mass of thigh, $r_{2}$ length to center of mass of calf, $g$ the gravitational acceleration, $\beta_{1}$ hip coefficient of friction and $\beta_{2}$ knee friction coefficient. The measurements were taken for a male person $1.77 \mathrm{~m}$ tall and $80 \mathrm{~kg}$, the mass of the thigh segment and the calf next to the center of mass of each one was taken according to the synthesis of classic anthropometry works presented by Winter [14]. Replacing the values of the constants of Table 1, in Eqs. (8) and (9), we obtain,

$$
\begin{aligned}
\tau_{1} & =31.72554 \sin \theta_{1}+0.27621 \dot{\theta}_{2}^{2} \sin \left(\theta_{1}-\theta_{2}\right)+0.27621 \ddot{\theta}_{2} \cos \left(\theta_{1}-\theta_{2}\right)+ \\
& +2.288 \dot{\theta}_{1}+1.0575 \ddot{\theta}_{1} \\
& \tau_{2}=6.021378 \sin \theta_{2}-0.27621 \dot{\theta}_{1}^{2} \sin \left(\theta_{1}-\theta_{2}\right)+0.27621 \ddot{\theta}_{1} \cos \left(\theta_{1}-\theta_{2}\right)+ \\
& +0.175 \dot{\theta}_{2}+0.101277 \ddot{\theta}_{2}
\end{aligned}
$$

here, Eqs. (10) and (11), represent a system of coupled partial differential equations.

\subsection{Matrix representation in state variables}

The matrix representation of the dynamic model is given by [15]

$$
M(\theta) \ddot{\theta}+C(\theta, \dot{\theta}) \dot{\theta}+G(\theta)=\tau,
$$

then, we can rewrite the system of Eqs. (12) like this,

$$
\begin{aligned}
& {\left[\begin{array}{cc}
m_{1} r_{1}^{2}+m_{2} l_{1}^{2} & m_{2} l_{1} r_{2} \cos \left(\theta_{1}-\theta_{2}\right) \\
m_{2} l_{1} r_{2} \cos \left(\theta_{1}-\theta_{2}\right) & m_{2} r_{2}^{2}
\end{array}\right]\left[\begin{array}{c}
\ddot{\theta}_{1} \\
\ddot{\theta}_{2}
\end{array}\right]+} \\
& {\left[\begin{array}{cc}
\beta_{1} & m_{2} l_{1} r_{2} \dot{\theta}_{2} \sin \left(\theta_{1}-\theta_{2}\right) \\
-m_{2} l_{1} r_{2} \dot{\theta}_{1} \sin \left(\theta_{1}-\theta_{2}\right) & \beta_{2}
\end{array}\right]\left[\begin{array}{c}
\dot{\theta}_{1} \\
\dot{\theta}_{2}
\end{array}\right]+} \\
& {\left[\begin{array}{c}
\left(m_{1} r_{1}+m_{2} l_{1}\right) g \sin \theta_{1} \\
m_{2} r_{2} g \sin \theta_{2}
\end{array}\right]=\left[\begin{array}{c}
\tau_{1} \\
\tau_{2}
\end{array}\right]}
\end{aligned}
$$

The state variables of a system are generally critical variables, of interest to be controlled, observed or that have an important relevance in the system. It is essential to keep 
in mind that these variables may not be accessible to the user; that is, they cannot be measured with sensors, so in these cases the use of state observers is necessary. In the case of leg prostheses, four variables are critical, which are the position and angular velocity of each link. For these four state variables the use of an observer is not necessary, since physically they can be measured with encoders and there is the concept of classical mechanics that the position is the integral of velocity, and velocity is the integral of acceleration.

Clearing the accelerations, we get,

$$
\begin{gathered}
\ddot{\theta}_{1}=\frac{\tau_{1}-m_{2} l_{1} r_{2} \dot{\theta}_{2}^{2} \sin \left(\theta_{1}-\theta_{2}\right)-\beta_{1} \dot{\theta}_{1}-\left(m_{2} l_{1}+m_{1} r_{1}\right) g \sin \theta_{1}}{m_{2} l_{1}^{2}+m_{1} r_{1}^{2}-m_{2} l_{1}^{2} \cos ^{2}\left(\theta_{1}-\theta_{2}\right)}- \\
-\frac{l_{1} \cos \left(\theta_{1}-\theta_{2}\right)\left[m_{2} l_{1} r_{2} \dot{\theta}_{1}^{2} \sin \left(\theta_{1}-\theta_{2}\right)+\tau_{2}-\beta_{2} \dot{\theta}_{2}-m_{2} r_{2} g \sin \theta_{2}\right]}{m_{2} r_{2} l_{1}^{2}+m_{1} r_{1}^{2} r_{2}-m_{2} r_{2} l_{1}^{2} \cos ^{2}\left(\theta_{1}-\theta_{2}\right)}, \\
\ddot{\theta}_{2}=\frac{\left(m_{1} r_{1}^{2}+m_{2} l_{1}^{2}\right)\left[m_{2} l_{1} r_{2} \dot{\theta}_{1}^{2} \sin \left(\theta_{1}-\theta_{2}\right)+\tau_{2}-\beta_{2} \dot{\theta}_{2}-m_{2} r_{2} g \sin \theta_{2}\right]}{m_{2}^{2} l_{1}^{2} r_{2}^{2}+m_{1} m_{2} r_{1}^{2} r_{2}^{2}-m_{2}^{2} l_{1}^{2} r_{2}^{2} \cos ^{2}\left(\theta_{1}-\theta_{2}\right)}+ \\
+\frac{l_{1} \cos \left(\theta_{1}-\theta_{2}\right)\left[m_{2} l_{1} r_{2} \dot{\theta}_{2}^{2} \sin \left(\theta_{1}-\theta_{2}\right)-\tau_{1}+\beta_{1} \dot{\theta}_{1}+\left(m_{1} r_{1}+m_{2} l_{1}\right) g \sin \theta_{1}\right]}{m_{2} r_{2} l_{1}^{2}+m_{1} r_{1}^{2} r_{2}-m_{2} r_{2} l_{1}^{2} \cos ^{2}\left(\theta_{1}-\theta_{2}\right)} .
\end{gathered}
$$

Because the theoretical model obtained is nonlinear, it is required to linearize it for the design of the controller in state space. The linearization of the system was performed around its unstable equilibrium point using Taylor's series, where only the first term of the series is taken since it is the linear term. The values of the state variables taken for the linear approximation are $(0,0,0,0)$, these values are due to the fact that the speeds must be zero for the system to remain in the desired position. Based on the above, the calculation of the linear model will be based on the state variables, which have the form,

$$
\mathbf{x}=\left[\begin{array}{c}
\mathbf{x}_{1} \\
\mathbf{x}_{2} \\
\mathbf{x}_{3} \\
\mathbf{x}_{4}
\end{array}\right]=\left[\begin{array}{c}
\theta_{1} \\
\dot{\theta}_{1} \\
\theta_{2} \\
\dot{\theta}_{2}
\end{array}\right]=\left[\begin{array}{c}
\theta_{1} \\
\omega_{1} \\
\theta_{2} \\
\omega_{2}
\end{array}\right]
$$

and the equations of state are,

$$
\dot{\mathbf{x}}=\left[\begin{array}{c}
\dot{\mathbf{x}}_{1} \\
\dot{\mathbf{x}}_{2} \\
\dot{\mathbf{x}}_{3} \\
\dot{\mathbf{x}}_{4}
\end{array}\right]=\left[\begin{array}{c}
\dot{\theta}_{1} \\
\ddot{\theta}_{1} \\
\dot{\theta}_{2} \\
\ddot{\theta}_{2}
\end{array}\right]=\left[\begin{array}{c}
\dot{\theta}_{1} \\
\dot{\omega}_{1} \\
\dot{\theta}_{2} \\
\dot{\omega}_{2}
\end{array}\right]
$$

To reduce the derivatives of the differential equations by one degree Eqs. (14) and (15), which represent the dynamic solution of the system, we define the following vectors of state, $\omega_{1}=\dot{\theta}_{1}, \omega_{2}=\dot{\theta}_{2}, \dot{\omega}_{1}=\ddot{\theta}_{1}, \dot{\omega}_{2}=\ddot{\theta}_{2}$, which physically represent the angular velocities and accelerations of both links. Once the state vectors have been defined, the Eqs. (14) and (15), can be rewritten as follows, 


$$
\dot{\mathbf{x}}_{1}=\mathbf{x}_{2}
$$

$$
\begin{aligned}
\dot{\mathbf{x}}_{2}=\dot{\omega}_{1} & =\frac{\tau_{1}-m_{2} l_{1} r_{2} \omega_{2}^{2} \sin \left(\theta_{1}-\theta_{2}\right)-\beta_{1} \omega_{1}-\left(m_{2} l_{1}+m_{1} r_{1}\right) g \sin \theta_{1}}{m_{2} l_{1}^{2}+m_{1} r_{1}^{2}-m_{2} l_{1}^{2} \cos ^{2}\left(\theta_{1}-\theta_{2}\right)}- \\
& -\frac{l_{1} \cos \left(\theta_{1}-\theta_{2}\right)\left[m_{2} l_{1} r_{2} \omega_{1}^{2} \sin \left(\theta_{1}-\theta_{2}\right)+\tau_{2}-\beta_{2} \omega_{2}-m_{2} r_{2} g \sin \theta_{2}\right]}{m_{2} r_{2} l_{1}^{2}+m_{1} r_{1}^{2} r_{2}-m_{2} r_{2} l_{1}^{2} \cos ^{2}\left(\theta_{1}-\theta_{2}\right)}
\end{aligned}
$$

$$
\dot{\mathbf{x}}_{3}=\mathbf{x}_{4}
$$

$$
\begin{aligned}
\dot{\mathbf{x}}_{4}=\dot{\omega}_{2} & =\frac{\left(m_{1} r_{1}^{2}+m_{2} l_{1}^{2}\right)\left[m_{2} l_{1} r_{2} \omega_{1}^{2} \sin \left(\theta_{1}-\theta_{2}\right)+\tau_{2}-\beta_{2} \omega_{2}-m_{2} r_{2} g \sin \theta_{2}\right]}{m_{2}^{2} l_{1}^{2} r_{2}^{2}+m_{1} m_{2} r_{1}^{2} r_{2}^{2}-m_{2}^{2} l_{1}^{2} r_{2}^{2} \cos ^{2}\left(\theta_{1}-\theta_{2}\right)}+ \\
& +\frac{l_{1} \cos \left(\theta_{1}-\theta_{2}\right)\left[m_{2} l_{1} r_{2} \omega_{2}^{2} \sin \left(\theta_{1}-\theta_{2}\right)-\tau_{1}+\beta_{1} \omega_{1}+\left(m_{1} r_{1}+m_{2} l_{1}\right) g \sin \theta_{1}\right]}{m_{2} r_{2} l_{1}^{2}+m_{1} r_{1}^{2} r_{2}-m_{2} r_{2} l_{1}^{2} \cos ^{2}\left(\theta_{1}-\theta_{2}\right)}
\end{aligned}
$$

The following border conditions are assumed,

$$
\begin{gathered}
\theta_{1}=0 \rightarrow \omega_{1}=0 \\
\theta_{2}=0 \rightarrow \omega_{2}=0,
\end{gathered}
$$

taking as a reference the upright position of a person on both feet. The equilibrium points suggested by experimental physics [16], are $(0,0,0,0),(0, \pi, 0,0),(\pi, \pi, 0,0)$, $(\pi, 0,0,0)$. These points are where the force of gravity, $g$, does not influence the movement and like the acceleration, $\ddot{\theta}_{1}=\ddot{\theta}_{2}=0$, the masses of the two pendulums never change places. Now as $\sin (\pi)=\sin (0)=\sin (-\pi)=0=\dot{\mathbf{x}}_{2}=\dot{\mathbf{x}}_{4}=0$. Points $(0, \pi, 0,0),(\pi, \pi, 0,0),(\pi, 0,0,0)$, are unstable. Gravity is what ensures the instability of these points. The reason is that in a neighborhood of any of these points, the force of gravity will involve a change in the acceleration of the pendulum centers of mass, $\dot{\mathbf{x}}_{2}$, $\dot{\mathbf{x}}_{4}$, and this change implies a change in position, the change is in the direction of the set of points such that $\mathbf{x}_{1}=\mathbf{x}_{3}=0$. This implies that for every neighborhood of the equilibrium points there is an orbit that comes out of that neighborhood, and therefore the points are unstable.

\subsection{Graphical Analysis}

In this section we analiced the solutions for different points. 


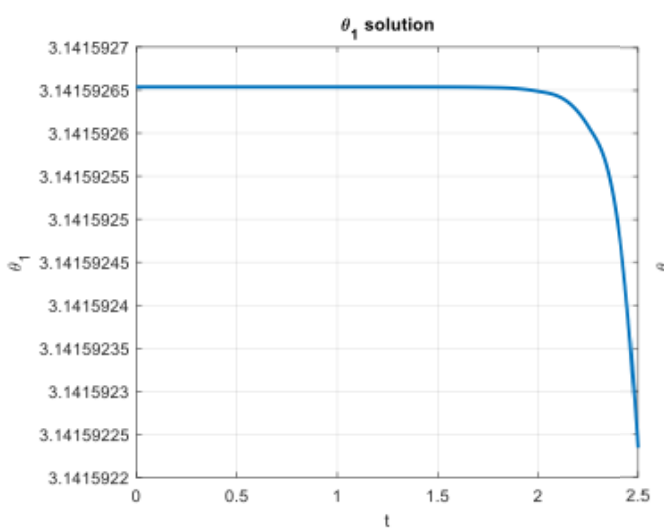

Figure 2. Solution $\theta_{1}$ for $(\pi, 0,0,0)$

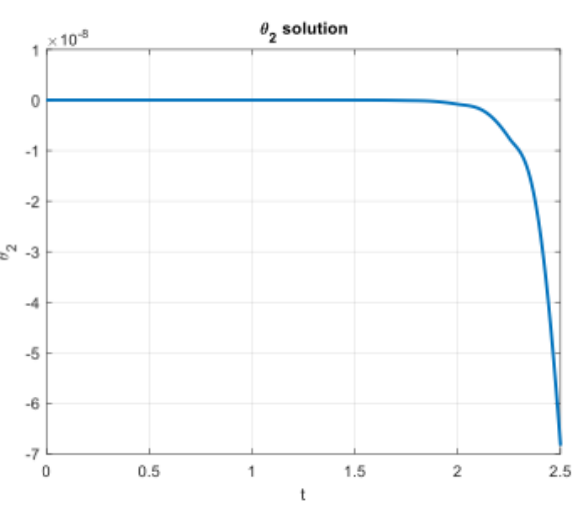

Figure 3. Solution $\theta_{2}$ for $(\pi, 0,0,0)$

At this point the hip angle at $180^{\circ}$ is evaluated, since the leg is not able to rotate to this position, the natural response of the system is unstable as can be seen in the Figure 2 and the Figure 3.

Analyzing the point of operation $(\pi / 9,0,2 \pi / 2.5,0)$ of the swing phase, which occurs between the take-off of the foot and the middle phase of the swing, where the hip is in neutral position and the knee rotates $20^{\circ}$ with an angular velocity of $2.51[\mathrm{rad} / \mathrm{s}]$, based on the cycle period, the following numerical solution is obtained.

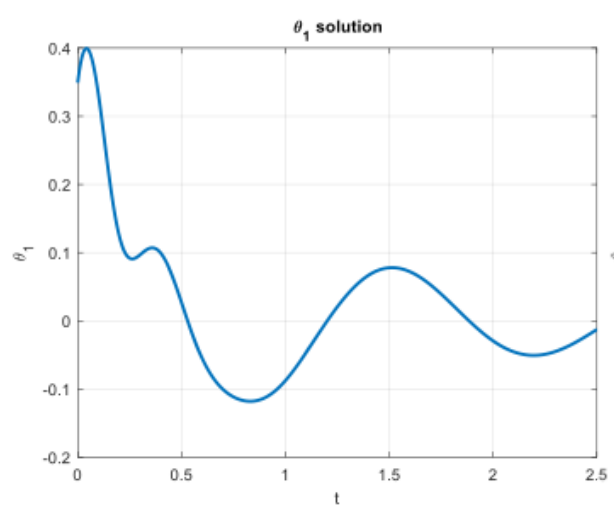

Figure 4. Solution $\theta_{1}$ for $(\pi / 9,0,2 \pi / 2.5,0)$

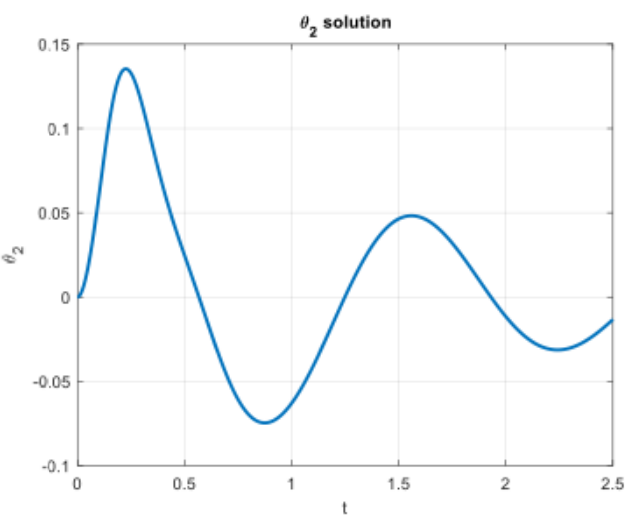

Figure 5. Solution $\theta_{2}$ for $(\pi / 9,0,2 \pi / 2.5,0)$ 


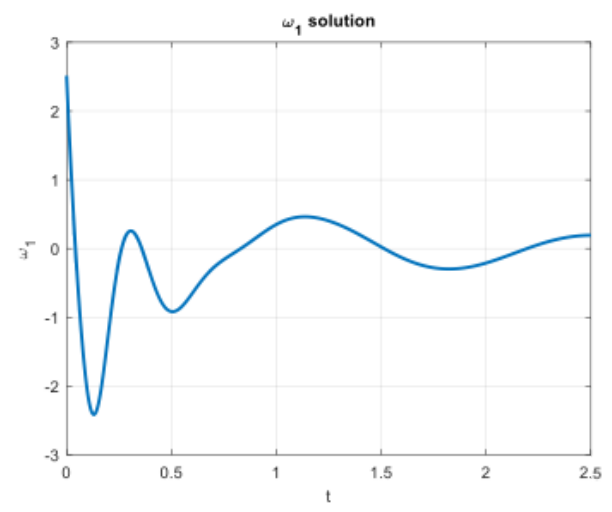

Figure 6. Solution $\dot{\theta}_{1}$ for $(\pi / 9,0,2 \pi / 2.5,0)$

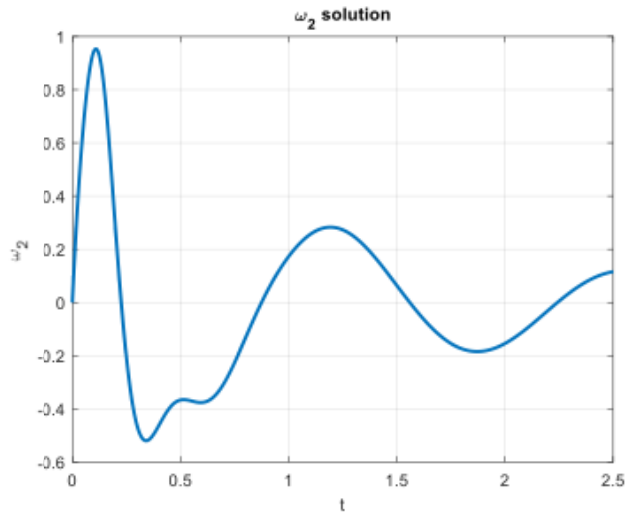

Figure 7. Solution $\dot{\theta}_{2}$ for $(\pi / 9,0,2 \pi / 2.5,0)$

It can be seen that in the Figure 4 and the Figure 5, the graph starts from the point designated $\pi / 9$ for $\theta_{1}$, oscillating in an acceptable working range for the angles of rotation performed by the hip, as well as the angular velocity of the hip $\dot{\theta}_{1}$ that starts at $2.51[\mathrm{rad} / \mathrm{s}]$ as shown in Figure 6. Furthermore, even though the knee angle $\theta_{2}$ and the angular velocity $\dot{\theta}_{2}$ is zero, it is observed how these vary during the 2.5 seconds of the gait cycle, which indicates that the equations of motion are coupled, that is, one depends on the other, as can be seen in Figure 7.

\subsection{Linealization}

An approximate linearization of the system was performed using Taylor's series, in which the selected operating points are $(0,0,0,0)$. For the system [17]-[18],

$$
\begin{aligned}
\dot{\mathbf{x}} & =A \mathbf{x}+B u \\
y & =\widetilde{C} \mathbf{x},
\end{aligned}
$$

where, $A, B$ y $\widetilde{C}$, are the Jacobian matrices of the system and are generally calculated and take the form,

$$
\begin{gathered}
A=\left[\begin{array}{cccc}
0 & 1 & 0 & 0 \\
-\frac{g\left(m_{1} r_{1}+m_{2} l_{1}\right)}{m_{1} r_{1}^{2}} & -\frac{\beta_{1}}{m_{1} r_{1}^{2}} & \frac{m_{2} g l_{1}}{m_{1} r_{1}^{2}} & \frac{l_{1} \beta_{2}}{m_{1} r_{2} r_{1}^{2}} \\
0 & 0 & 0 & 1 \\
\frac{m_{2} g l_{1}^{2}+m_{1} g r_{1} l_{1}}{m_{1} r_{2} r_{1}^{2}} & \frac{l_{1} \beta_{1}}{m_{1} r_{2} r_{1}^{2}} & -\frac{g\left(m_{1} r_{1}^{2}+m_{2} l_{1}^{2}\right)}{m_{1} r_{2} r_{1}^{2}}-\frac{\beta_{2}\left(m_{1} r_{1}^{2}+m_{2} l_{1}^{2}\right)}{m_{1} m_{2} r_{1}^{2} r_{2}^{2}}
\end{array}\right] \\
B=\left[\begin{array}{c}
0 \\
\frac{1}{m_{1} r_{1}^{2}} \\
0 \\
0 \\
\frac{m_{1} r_{1}^{2}+m_{2} l_{1}^{2}}{m_{1} m_{2} r_{1}^{2} r_{2}^{2}}
\end{array}\right]
\end{gathered}
$$




$$
\widetilde{C}=\left[\begin{array}{llll}
1 & 0 & 0 & 0 \\
0 & 0 & 1 & 0
\end{array}\right]
$$

Replacing the value of the constants of the Table 1, in Eqs. (22), (23), and (24), the following linearized matrices of the system are obtained,

$$
\begin{aligned}
& A=\left[\begin{array}{cccc}
0 & 1 & 0 & 0 \\
-104.2917 & -7.5214 & 53.9840 & 1.5689 \\
0 & 0 & 0 & 1 \\
284.4320 & 20.5128 & -206.6837 & -6.0069
\end{array}\right] \\
& B=\left[\begin{array}{c}
0 \\
3.2873 \\
0 \\
34.3250
\end{array}\right] \\
& \widetilde{C}=\left[\begin{array}{llll}
1 & 0 & 0 & 0 \\
0 & 0 & 1 & 0
\end{array}\right]
\end{aligned}
$$

Given the linearized matrices Eqs. (25), (26) and (27), it can be seen that the system is a SIMO case, where there is one input ( 1 column in matrix $B$ ) and two outputs ( 2 rows in matrix $\widetilde{C}$ ), from the above the transfer matrix can be obtained,

$$
M . T=\frac{1}{s^{4}+13.5 s^{3}+324 s^{2}+627.4 s+6200.6}\left[\begin{array}{c}
3.3 s^{2}+73.6 s+2532.4 \\
34.3 s^{2}+325.6 s+4514.8
\end{array}\right]
$$

The transfer matrix Eq. (28), is defined as the relationship between the inputs and outputs of the system, in the denominator is the characteristic polynomial, which for this case is fourth degree, that is, the system has four poles, this number of poles is the same that must have the desired poles to control the system.

\section{Dynamic system control}

A state feedback controller was designed due to its good performance in the face of modeling inaccuracies or disturbances. However, before designing the controller, the controllability and observability of the system must be verified, this is done by calculating the controllability matrix $Q=\left[B A B A^{2} B A^{3} B\right]$ and the observability matrix 


$$
\mathcal{O}:=\left[\begin{array}{c}
\widetilde{C} \\
\widetilde{C} A \\
\widetilde{C} A^{2} \\
\vdots \\
\widetilde{C} A^{n-1}
\end{array}\right]=n
$$

to verify the controllability and observability each of the matrices must have rank $n$ [18], and effectively for this case, each matrix has a rank equal to 4. for our case we have,

$$
Q=\left[\begin{array}{cccc}
0 & 3.2873 & 29.1289 & 1073.4 \\
3.2873 & 29.1289 & 1073.4 & -26020 \\
0 & 34.325 & -138.7538 & -4728.4 \\
34.325 & -138.7538 & -4728.4 & 87384
\end{array}\right]
$$

and

$$
\mathcal{O}:=\left[\begin{array}{cccc}
1 & 0 & 0 & 0 \\
0 & 0 & 1 & 0 \\
0 & 1 & 0 & 0 \\
0 & 0 & 0 & 1 \\
-104.2917 & -7.5214 & 53.9840 & 1.3689 \\
284.4320 & 20.5128 & -206.6837 & -6.0069 \\
1230.7 & -15.5373 & -730.3088 & 32.7590 \\
3847.9 & 6.9216 & 2348.9 & -138.4177
\end{array}\right]=\operatorname{Rank}(\mathcal{O})=4
$$

The design of the controller was carried out using the pole allocation technique, the desired poles of the system are placed under the criterion of settlement time $t_{s}=4 T$, (where $T=2.5$ seconds the duration of the running cycle), therefore, the settling time is 10 seconds and the overshoot, which is defined by the maximum amount that the response exceeds the steady state value, in this case, you want to attenuate this as much as possible parameter, this is why an overshoot less than $5 \%$ is defined. With the two previous parameters the dominant poles of the controller are determined by means of the equations,

$$
\begin{gathered}
\xi=\frac{\ln \left(\frac{O V}{100}\right)}{\sqrt{\pi^{2}+\ln \left(\frac{O V}{100}\right)^{2}}}=\frac{\ln \left(\frac{5}{100}\right)}{\sqrt{\pi^{2}+\ln \left(\frac{5}{100}\right)^{2}}}=0.69 \\
\omega_{n}=\frac{4}{\xi t_{s}}=\frac{4}{(0.69)(10)}=0.57
\end{gathered}
$$

where, $O V$, is the overshoot value, ts is the settlement time, then the values obtained from Eqs. (31) and (32), are replaced in the equation, 


$$
s^{2}+2 \xi \omega_{n} s+\omega_{n}^{2}=s^{2}+0.8 s+0.33
$$

solving the quadratic equation, we know the dominant poles of the controller

$$
\begin{aligned}
& \mu_{1}=-0.4+0.4195 i, \\
& \mu_{2}=-0.4-0.4195 i .
\end{aligned}
$$

Since the system is of order 4, that is, it has four poles, this is deduced from the Eq. (28), four poles must be defined for the controller, from Eq. (33), two dominant poles were obtained, and the remaining two poles are insignificant, which are located 5 to 10 times farther than the dominant poles $\mu_{3}=-50$ and $\mu_{4}=-45$. Feedback gains are determined using Ackerman's formula [18],

$$
K=\left[\begin{array}{llll}
0 & 0 & 0 & 1
\end{array}\right] Q^{-1} \phi(A),
$$

where, $\phi(A)=A^{4}+\alpha_{1} A^{3}+\alpha_{2} A^{2}+\alpha_{3} A+\alpha_{1} \mathbf{I}$. The characteristic equation is given by,

$$
\begin{aligned}
|s-A+B K| & =|s \mathbf{I}-A| \\
& =\left(s-\mu_{1}\right)\left(s-\mu_{2}\right)\left(s-\mu_{3}\right)\left(s-\mu_{4}\right) \\
& =s^{4}+\alpha_{1} s^{3}+\alpha_{2} s^{2}+\alpha_{3} A+\alpha_{4},
\end{aligned}
$$

in our case,

$(s-0.4+0.4195 i)(s-0.4-0.4195 i)(s-50)(s-45)=s^{4}+95.8 s^{3}+2326.3 s^{2}+1831.9 s+756$

to get feedback gains

$$
K=\left[\begin{array}{llll}
-105.3237 & -9.8608 & 57.8717 & 3.3412
\end{array}\right]
$$

here, the Eq. (38), there is the controller gain vector, where $K_{1}=-105.3237$ is the state feedback gain $\mathbf{x}_{1}, K_{2}=-9.8608$ is the state gain $\mathbf{x}_{2}, K_{3}=57.8717$ is the state gain $\mathbf{x}_{3}$ and $K_{4}=3.3412$ is the gain of the status $\mathbf{x}_{4}$.

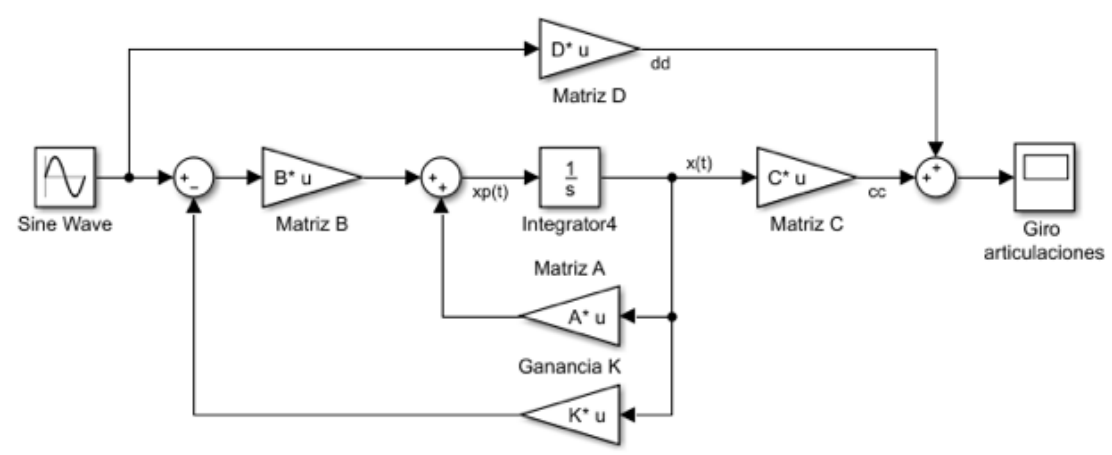

Figure 8. Block diagram with controller 
The Figure 8, shows the block diagram of the system, where the matrices $A, B, \widetilde{C}$ previously described are entered respectively, additionally, the controller feedback with the gain vector Eq. (38), the result of the controller simulation is shown in Figure 9.

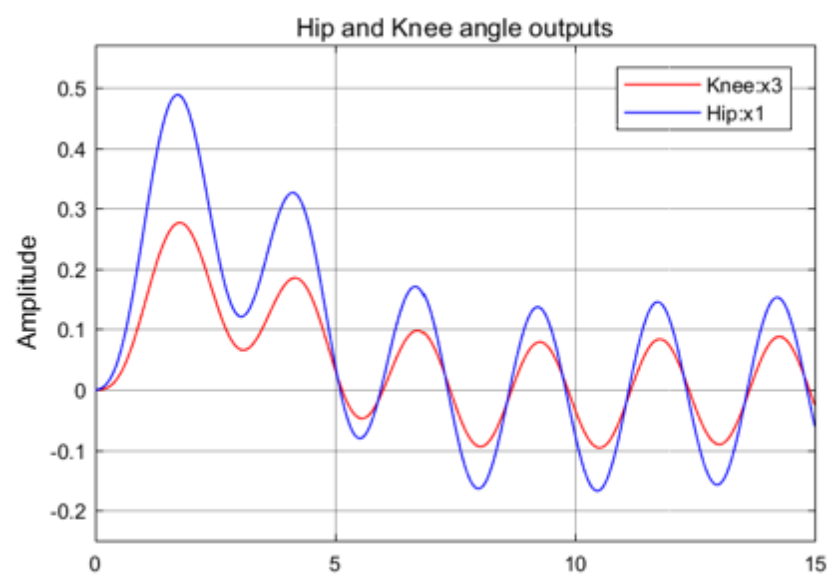

Figure 9. Hip and knee exit angles.

In the Figure 9, shows the response of the hip $\left(\theta_{1}\right)$ and knee $\left(\theta_{2}\right)$ angles with the controller to a sinusoidal input, where it can be seen that the hip joint has a greater amplitude with respect to the knee Since the hip has a wider turning range than the knee. In addition, it is shown how each of the two outputs try to follow the sinusoidal input until both stabilize in a certain time, keeping the input signal within the operating range of each one of them.

\section{Conclusions}

The human gait model resembles the mechanical behavior of a double pendulum, the equations of motion are obtained by means of the Euler-Lagrange formalism based on the analysis of energies, since this allows to obtain a dynamic model of the system, these equations they are linearized using the Taylor series approximation in order to design and implement a state feedback control system, which is based on a design in state space. As can be seen in Figure 9, the controller responds satisfactorily in such a way that it satisfies the variations of the angles of both the hip and the knee, following the input signal, giving good results to the pole assignment criterion and Ackerman's formula for obtaining feedback gains. On the other hand, this study allows us to define a dynamic model of human gait, which can be taken into account for the design of electromechanical devices such as prostheses and / or recovery devices.

\section{Acknowledgements}

P. A. Ospina-Henao, we are grateful for the permanent support of the Vicerrectoría Académica of Universidad Santo Tomás, with the project of the XII Internal Call for Research Projects, with initiation act 163 of February 05, 2020. 


\section{References}

[1] Erazo Bravo, M. y Mera Otoya, E., Diseño e implementación de un prototipo de prótesis transtibial con amortiguamiento activo mediante la investigación del comportamiento de materiales magnetoreológicos bajo diferentes campos magnéticos, Universidad De Las Fuerzas Armadas, Latacunga, Ecuador, 2017.

[2] Simonsen EB. Contributions to the understanding of gait control. Dan Med J. 2014; 61 (4): B4823.

[3] Valencia, Cesar H; Vellasco, Marley; Tanscheit, Ricardo and Figueiredo, Karla T. Magnetorheological Damper Control in a Leg Prosthesis Mechanical. Advances in Intelligent Systems and Computing. 2005. Pages 805-818.

[4] Arthur D. Kuo, J. Maxwell Donelan, Dynamic Principles of Gait and Their Clinical Implications, Physical Therapy, Volume 90, Issue 2, 1 February 2010, Pages 157-174.

[5] Hunt, M. et al. (2008). Measures of Frontal Plane Lower Limb Alignment Obtained from Static Radiographs and Dynamic Gait Analysis. Gait \& posture 27(4): 635-640.

[6] Schiehlena, W. and García-Vallejo D. Walking dynamics from mechanism models to parameter optimization, Procedia IUTAM 2 (2011), pp 199-211.

[7] González Míguez, Paula, author; Saló i Bru, Guillem, supervisor academic. Idelsohn Zielonka, Sebastian, academic supervisor; [et al.] Biomechanical analysis of gait using an analytical model of inverse dynamics in patients with altered sagittal balance of the spine. (121 pages). ISBN 9788449075650.

[8] Pratt, J. E. (2000). Exploiting Inherent Robustness and Natural Dynamics in the Control of Bipedal Walking Robots. Ph.D. Thesis, MIT.

[9] Pop, C. Bondgraphs Modeling and Model Evaluation of Human Locomotion Using Experimental Data. University Of Waterloo.

[10] Luengas LA. Static alignment model for transtibial prostheses. Bogotá: Pontificia Universidad Javeriana; 2016.

[11] Borghese NA, Bianchi L, Lacquaniti F. Kinematic determinants of human locomotion. J Physiol. 1996; 494 (3): 863-79.

[12] Goldstein. Classical Mechanics. Editorial Addison Wesley (1980).

[13] K. Ogata. Dinámica de Sistemas. Tercera ediciÃşn, Prentice Hall. México D.F., 1978.

[14] Winter, D. Biomechanics of human movement., John Wiley \& Sons, 1979.

[15] R. Kelly, V. Santibáñez. Control de Movimiento de Robots Manipuladores. PEARSON EDUCATION, S.A., Madrid, 2003, pp. 327.

[16] G. D. Quiroga, P. A. Ospina-Henao. Dynamics of damped oscillations: physical pendulum. European Journal of Physics, 2017.

[17] S. Domínguez, P. Campoy, J. M. Sebastián, A. Jiménez. Control en el Espacio de Estados. Segunda edición, PEARSON EDUCATION S.A., Madrid, 2006, pp. 440.

[18] K. Ogata. Ingeniería de Control Moderna. Tercera edición, PEARSON EDUCATION S.A. México D.F., 1998, pp. 1015. 